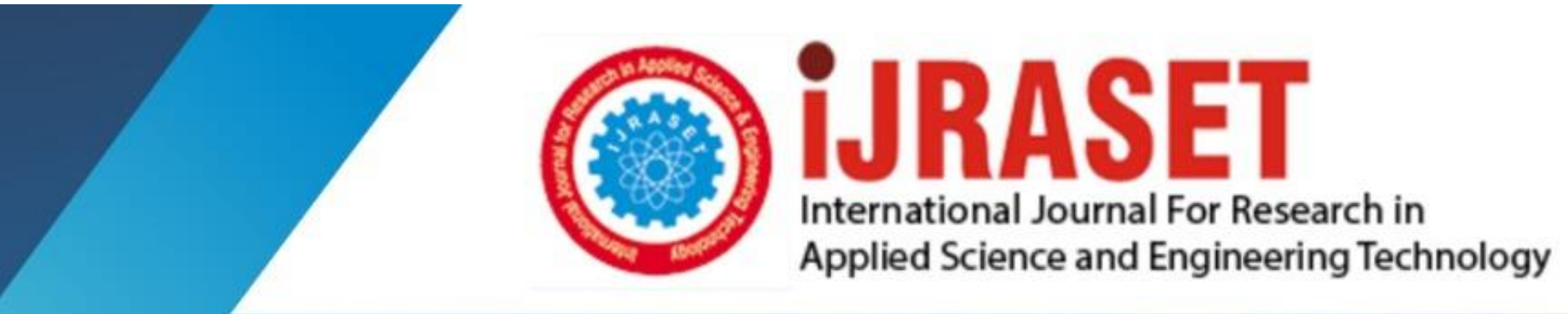

International Journal For Research in Applied Science and Engineering Technology

\title{
INTERNATIONAL JOURNAL FOR RESEARCH
}

IN APPLIED SCIENCE \& ENGINEERING TECHNOLOGY

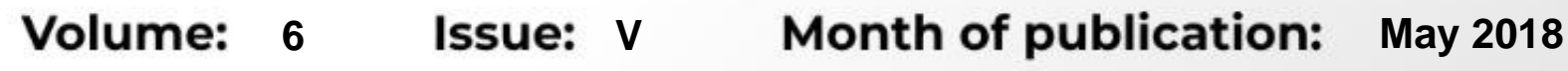
DOI: http://doi.org/10.22214/ijraset.2018.5451

\section{www.ijraset.com}

\section{Call: @08813907089｜Ｅ-mail ID: ijraset@gmail.com}




\title{
Vocal Based Online Examination
}

\author{
Varsha $\mathrm{V}^{1}$, Vinutha $\mathrm{S}^{2}$, Sahana $\mathrm{M} \mathrm{R}^{3}$, Sania Seemen $\mathrm{Y} \mathrm{K}^{4}$, Natesh $\mathrm{M}^{5}$, Ashwini C L \\ 1, 2, 3, 4 Department of CSE, Vidya Vardhaka College of Engineering, Mysore, India \\ ${ }^{5}$ Guide, Assistant Professor, Department of CSE, Vidya Vardhaka College of Engineering, Mysore, India \\ ${ }^{6}$ Guide, Senior Web Developer IT Department Technologica Solutions, Bengaluru, India
}

\begin{abstract}
The proposed paper is introducing an innovative and real-time economical beneficial technique that allows the users to hear the contents of text instead of reading them and speak the content instead of typing. It combines the concept of Text to Speech (TTS) and Speech to Text(STT) technologies. The paper propose framework consisting of web application with open source API's for TTS and STT. TTS conversion is a method reads Multilanguage text to voices using IVR-API. The STT convert voice to text of visually impaired people using Webkitspeechrecognition. This mobile application consists of two modules, admin module (register, login student and uploading exam paper) and student module (helps visually impaired people to interact with effectively through vocal interface).
\end{abstract}

Keywords: Text to Speech (TTS), Speech to Text(STT), IVR-API, Webkitspeechrecognition, Speech Synthesizer

\section{INTRODUCTION}

Speech Recognition recognizes the spoken words and phrases, and converts into a machine-readable format. By converting spoken audial into text, speech recognition technology allows users to control digital devices by talking instead of using conventional devices such as buttons, keystrokes, keyboards etc. Speech recognition is the mechanism on the speech or command of human speech to recognize and realize and react accordingly. It is grounded on the vocal sound as the research objective, it allows the mechanism to automatically recognize and realize human vocal language over speech signal processing and form recognition. The vocal recognition technology is the advanced tech that allows the machine to turn the voice signal into the applicable text or command through the process of identification and understanding. Speech recognition is a cross-disciplinary and includes a widespread range. Speech recognition is one of the best useful machineries for the disabled. When it is not imaginable or suitable to use a keyboard or a like devices the technology allows users not only to command their expressions but also to control devices. It has a very close connection with audibility, phonetics, semantics, information theory, and form recognition concept and neurobiology disciplines. With the quick growth of computer hardware and software and information technology, speech recognition technology is progressively becoming a vital technology in the computer information processing technology.

\section{PROBLEM STATEMENT}

Visually impaired or physically challenged students in the city find it difficult to find scribes when they have to write examinations in languages other than English. If the scribes does not make out at the last minute, the student needs to directly notify his/her school or college to arrange for another writer. Students have to bear the writer dropping out at the last minute

When we do not find someone, who is fluent in the language of instruction, it affects our performance in the examinations, Blind students cannot choose their scriber, and it's who are allocated to them by the third-party.

The scribers are also exchanged among the students, so they cannot improve a relationship with them. Sometimes there are confusions, which take their tax on results.

Examiners sit exams under the one grade lower system, by which a scribers one grade below the student sitting the exam helps read the questions and write the answers [1].

Additional main difficult students face is that their exams may bang with those of their scribers (for the period of the February or March). Even though the Ministry of Social Justice \& Empowerment in 2013 revised its guidelines and criteria for the scribes, a large number of students still struggle to get somebody to write for them.

\section{III.OBJECTIVE}

To overcome these problems, the paper based on speech recognition which would be beneficial for visually impaired students, physically disabled students and students undergone some injuries and accidents using open free resource available freely to develop and provide same to end users by doing survey on all resources available [2]. The answer format is sentence based instead of single letter base, support multi-language with efficiency [3]. 


\section{IV.METHODLOGY}

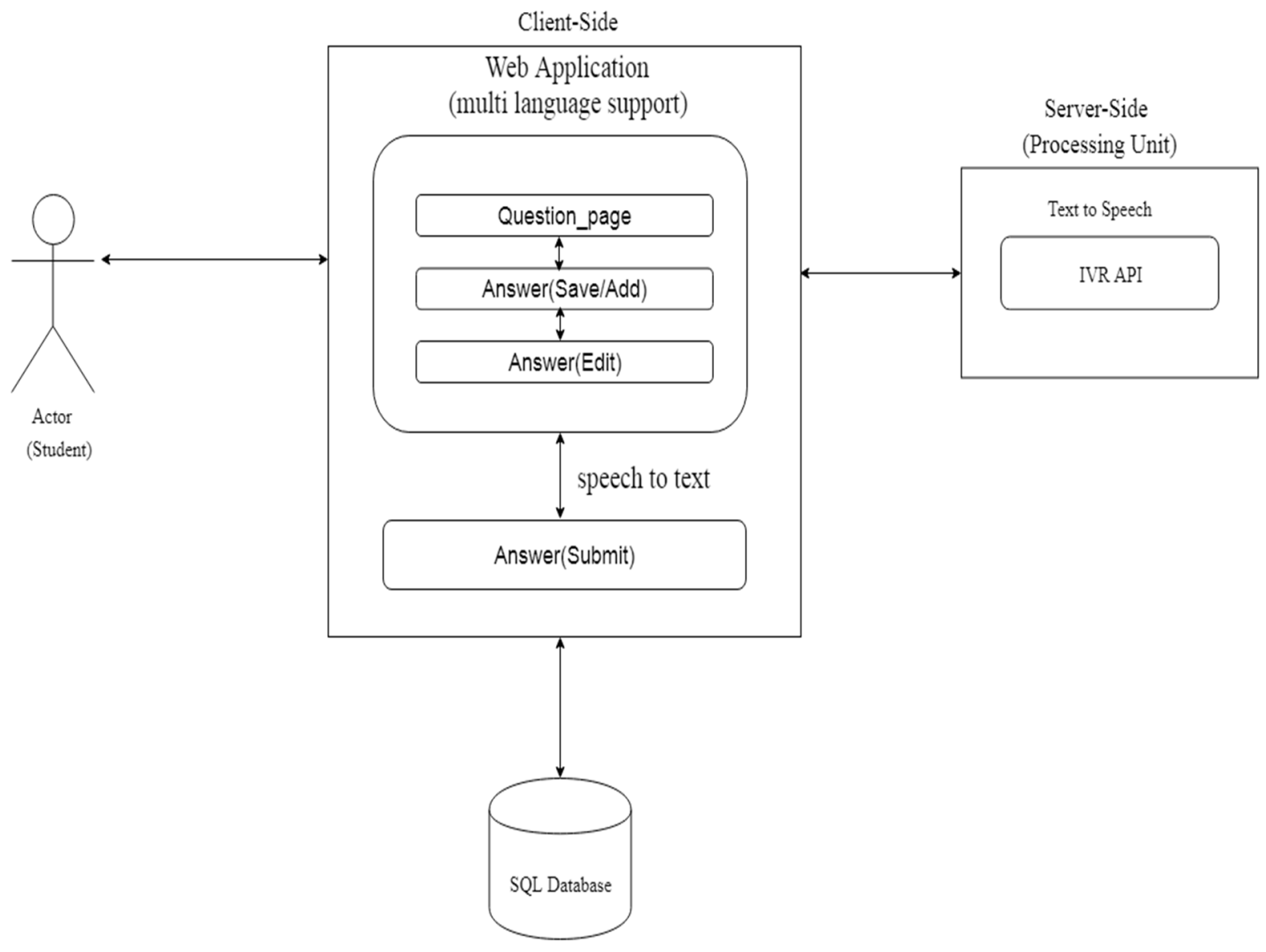

Fig. 1 System Architecture

The project consists of three main components as shown in above Fig. 1

\section{A. Graphic User Interface (GUI)}

The GUI is user/admin interaction web pages and even the client-side Speech to Text (STT) process is done here.

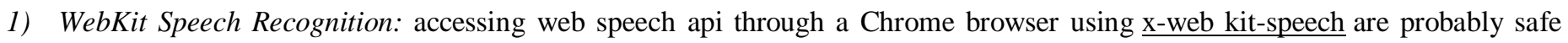
since it is supported by Google [4]. Let's web developers to afford speaking input and text output features in a web browser. Usually, these features aren't obtainable when using regular speech recognition or screen reader software. The API takes care of the secrecy of the users. Before letting the website to entree the speech by microphone, the user must clearly award permission.

\section{B. Processing Unit}

Its server-side Text to Speech (TTS) processing by using Interactive voice response (IVR), IVR API which cloud support and REST API $[5,6,7]$. IVR is a technology that lets a computer to work together with humans using speech and DTMF tones input. In telecommunications, IVR permits customers to interact with a company's cloud system via speech recognition, after which facilities can be queried through the IVR dialogue [5]. IVR methods can respond with dynamically generated voice to promote direct users on how to proceed.

IVR method installed in the network are sized to grip large call volumes and used for outbound calling, as IVR methods are more intelligent than many projecting diallers' methods. Using the IVR API api-key and user-id able to convert Multilanguage text to speech.

\section{Database}

The database is used to register students and submit the answer sheets of students. 


\section{RESULTS}

Once the user logins paper list (English/Hindi) will be shown and then the paper will be choosen.in the above Fig. 2, Hindi paper has been chosen.

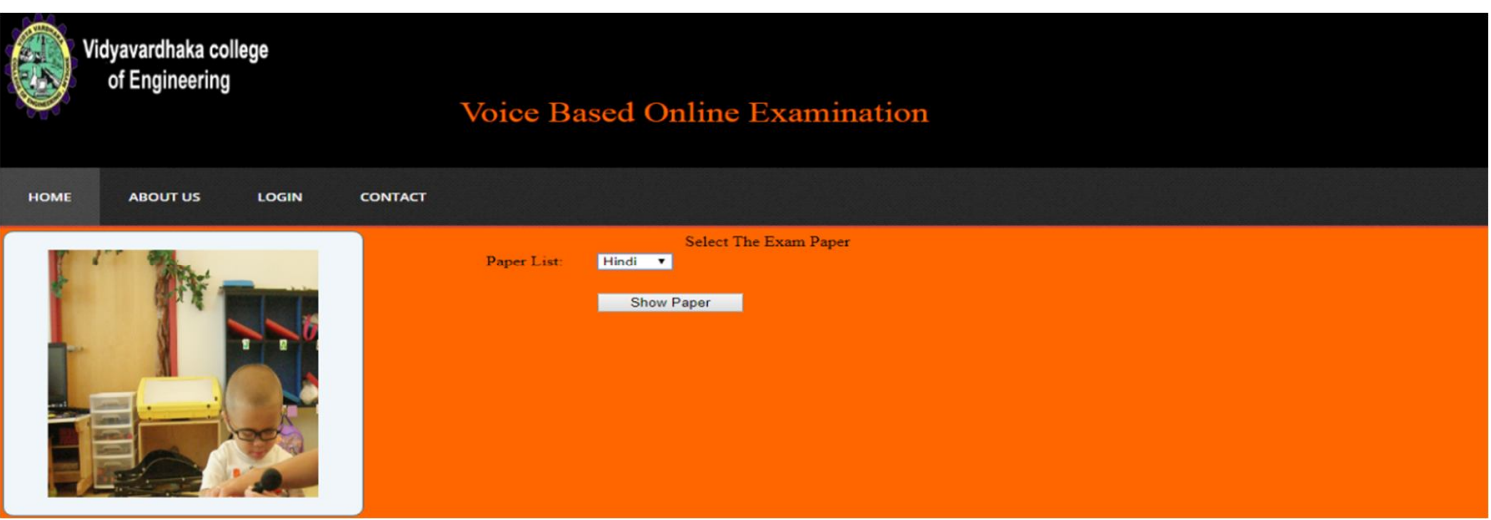

Fig. 2 Selecting exam paper

After selecting the subject, question paper of that selected subject will be displayed and it will be heard by the user later user chooses the question which he/she would like to answer. As shown in Fig.3, the user chosen question is first by saying pehala(Hindi)

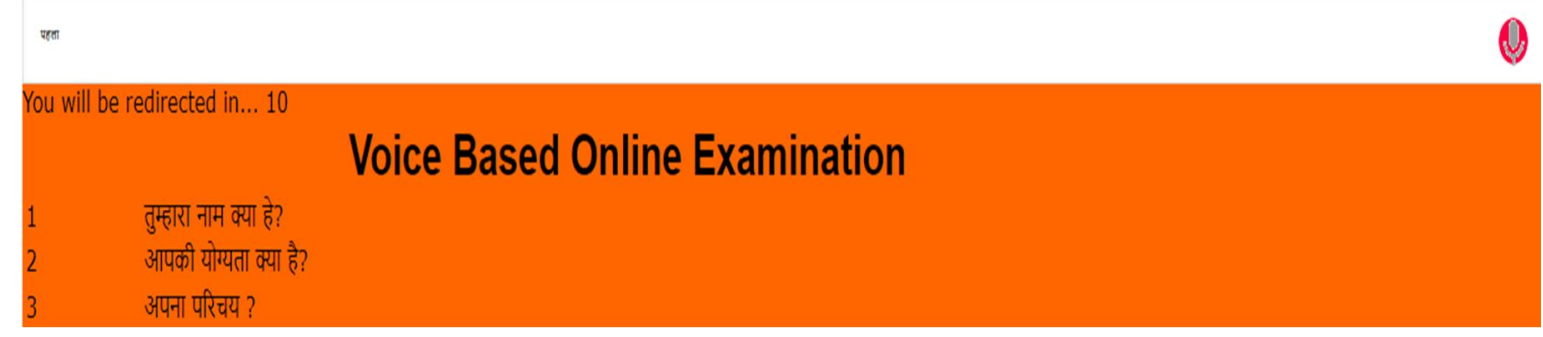

Fig. 3 choose question to answer

After choosing the question it will be directed to answer page. Where the user will answer for the chosen question. After answering the user will save/submit/add/edit the answer by saying save/Submit. As shown in Fig.4, the user answered the first question and saved the answer.

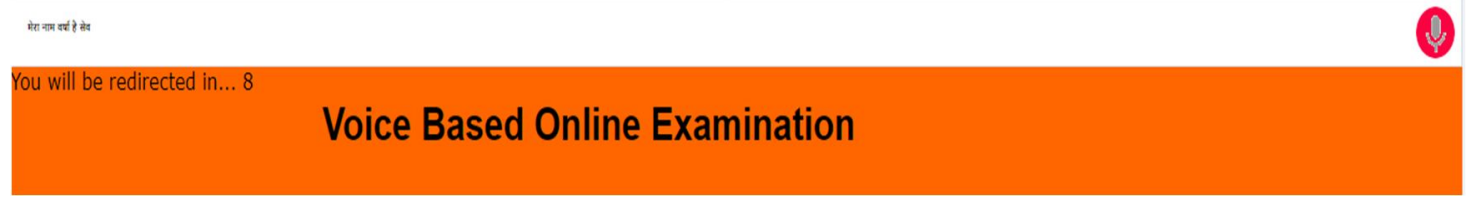

Fig. 4 Answer the question

Once the answer is saved it will be redirected to the question page. Once again, the user selects the question. If user choses to edit, he/she will hear the answer said by the user and ask for the sentence user likes to edit. As shown in Fig.5, the user is saying the sentence that is to be edited.

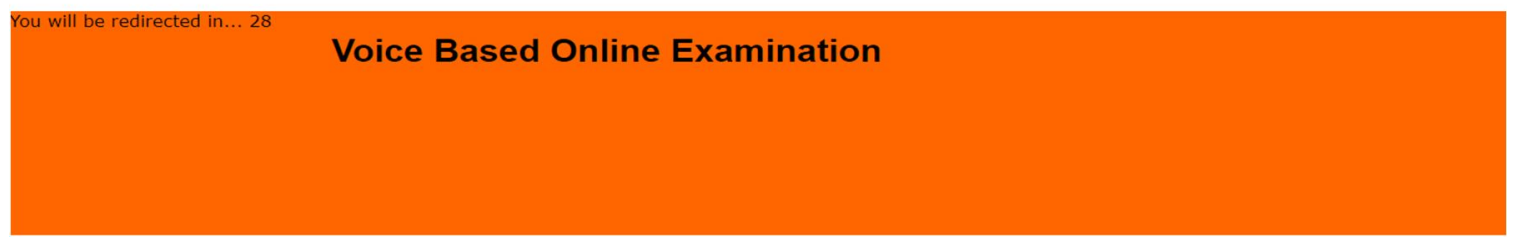

Fig. 5 Edit the answer 
Once the user finishes answering all the questions and if he does not wish to edit any answer, the user will say submit and the answers which has been answered by the user will be stored in the database. As shown in Fig.6, the user is saying submit to submit the answer.

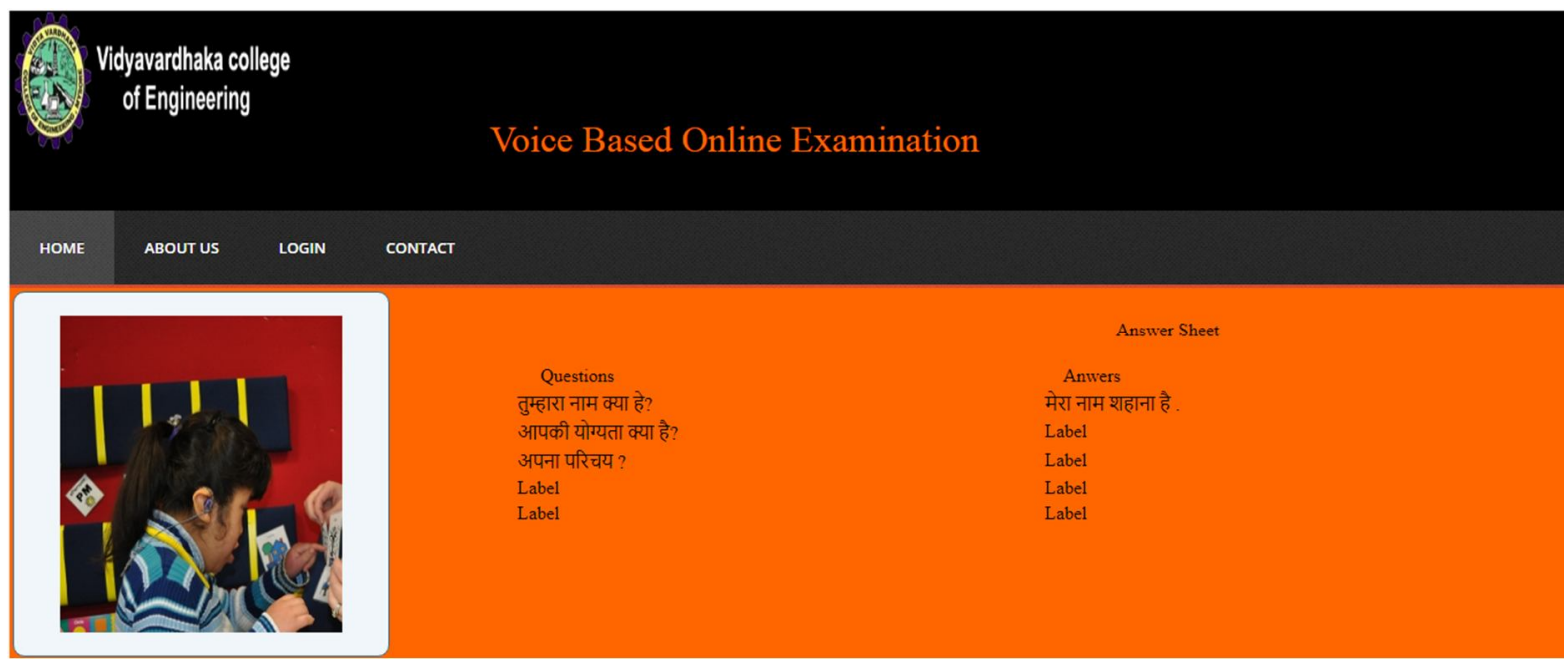

Fig. 6 Answers stored in database

Once the user submits the answers, the answers will be stored in the database and the submitted answer will be displayed in the page as shown in Fig.6. No further changes can be made for answers once submitted

\section{VI.CONCLUSIONS}

A user-friendly web application is developed for the beneficial of visually impaired students and handicapped students which provide reading and writing assistance using technologies like text to speech and speech to text application programming interfaces. The proposed paper would be a very useful for every blind people and physically challenged to admire their talent easily through online exam like other humans. In proposed paper will be going to deliver an entire application for physically challenged people which can provide an interactive interface. Examinee can easily give exam by giving easy voice commands. Thus, physically challenged people can easily give exam like a common man without much difficulty. In the future will try to advance the features like security, STT and TTS algorithms, efficiency etc., as per the collection of feedback and experts suggestions.

\section{REFERENCES}

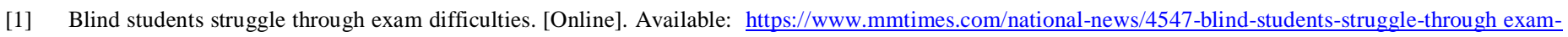
difficulties.html

[2] What are the top ten speech recognition APIs? [Online]. Available: https:/www.quora.com/What-are-the-top-ten-speech-recognition-APIs

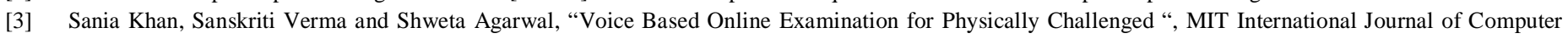
Science and Information Technology, Vol. 5, No. 2, August 2015, pp. 58-61 58 ISSN 2230-7621@MIT Publications

[4] Web Speech API. [Online]. Available: https://developer.mozilla.org/en-US/docs/Web/API/Web_Speech_API

[5] Indiantts. [Online]. Available: http://indiantts.com/IVR-API.html

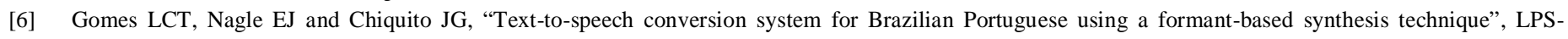
DECOM-FEEC-Unicamp.

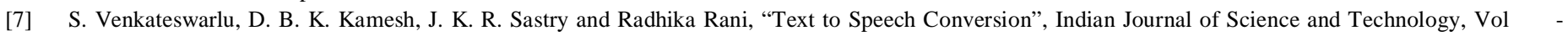
9(38), DOI: 10.17485/ijst/2016/v9i38/102967, October 2016. 
li $=$

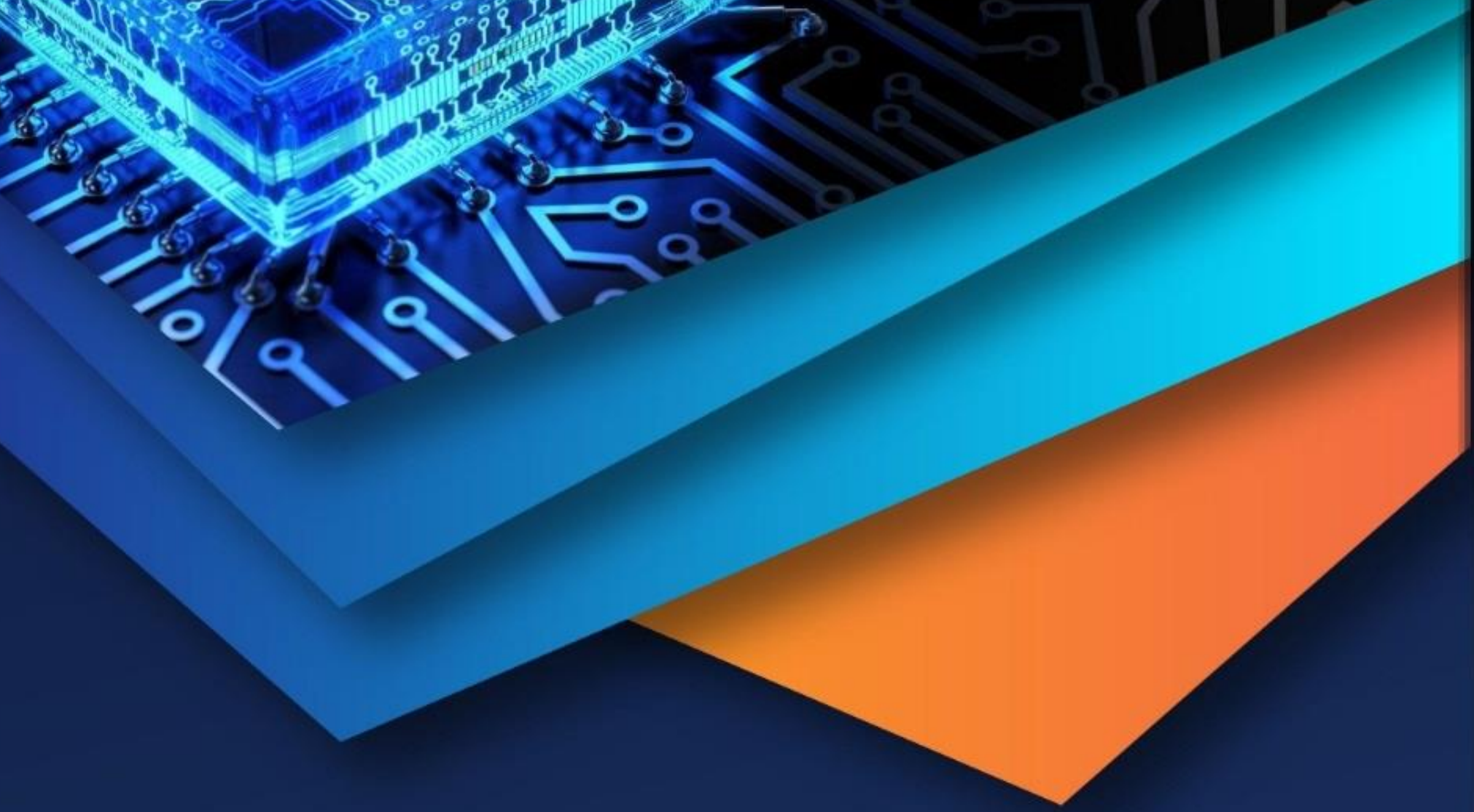

do: cross ref

10.22214/IJRASET

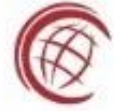

INDEX COPERNICUS

45.98

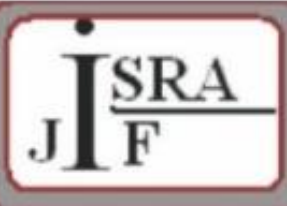

IMPACT FACTOR: 7.129

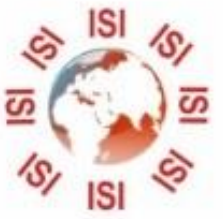

IMPACT FACTOR:

7.429

\section{INTERNATIONAL JOURNAL FOR RESEARCH}

IN APPLIED SCIENCE \& ENGINEERING TECHNOLOGY

Call : $08813907089 @\left(24^{*} 7\right.$ Support on Whatsapp) 